$\ll$ Review $\gg$

\title{
Molecular Bases for Seasonal Reproduction in Birds
}

\author{
Takashi Yoshimura \\ Division of Biomodeling, Graduate School of Bioagricultural Sciences, \\ Nagoya University, 464-8601, Japan
}

\begin{abstract}
Appropriate timing of various seasonal processes is crucial to the survival and reproductive success of animals inhabiting temperate regions. When seasonally breeding animals are subjected to annual changes in daylength, dramatic changes in neuroendocrine-gonadal activity ensue. However, the molecular mechanism of photoperiodic (or seasonal) time measurement (PTM) is not well understood for any organism living. Japanese quail is an excellent model for studying PTM because of the rapid and dramatic response to photoperiod. Here I describe recent progress in understanding the molecular mechanism of PTM in Japanese quail.
\end{abstract}

Key words : circadian clock, Japanese quail, photoperiodism, thyroid hormone

\section{Introduction}

Reproduction of many temperate-zone birds is under photoperiodic control. Although melatonin has an important role for the regulation of photoperiodic time measurement (PTM) in mammals, no effect of melatonin manipulations is observed in birds (Gwinner et al., 1997). Japanese quail is an appropriate model system for the investigation of the PTM because of the rapid and dramatic response to photoperiod (Follett and Sharp, 1969 ; Wada, 1979). Therefore, a considerable number of studies have been made on Japanese quail over the past few decades. Numerous reports have suggested that the mediobasal hypothalamus $(\mathrm{MBH})$, including the infundibular nucleus (IN), the inferior hypothalamic nucleus (IH), the median eminence (ME) and nucleus hypothalamicus posterior medialis (NHPM) is an important center controlling the PTM (Fig. 1). For example, lesions of the IN, the ME, or NHPM within the MBH

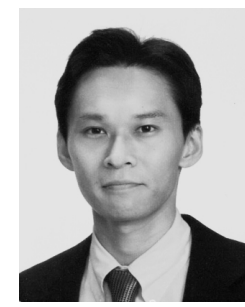

Takashi Yoshimura

1995 : M. Agr. (Animal Science) Nagoya University, Japan

1999 : Ph.D. (Agriculture) Nagoya University, Japan

Current position

Assistant Professor in Graduate School of Bioagricultral Sciences, Nagoya University

Area of research interests : Molecular mechanism of seasonal reproduction in vertebrates, Mechanism for ovulation-oviposition cycle in birds.

Received : June 17, 2004, Accepted : July 5, 2004

Corresponding author: Takashi Yoshimura, Ph.D. Division of Biomodeling, Graduate School of Bioagricultural Sciences, Nagoya University, Furo-cho, Chikusa-ku, Nagoya 464-8601, Japan

Tel \& Fax : +81-52-789-4056 E-mail : takashiy@agr.nagoya-u.ac.jp 


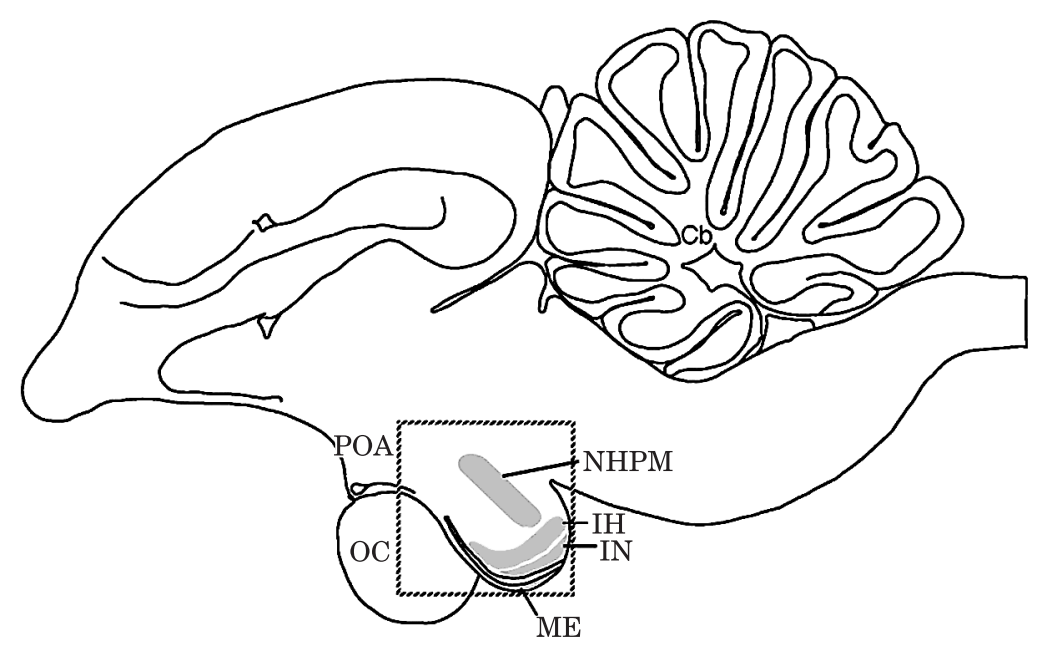

Fig. 1. The mediobasal hypothalamus (MBH) (dashed line) of Japanese quail. $\mathrm{Cb}$ : cerebellum ; IH : inferior hypothalamic nucleus ; IN : infundibular nucleus; ME : median eminence; NHPM : nucleus hypothalamicus posterior medialis; OC: optic chiasm; POA : preoptic area.

can block the rise of luteinizing hormone $(\mathrm{LH})$ and testicular growth under long photoperiods (Sharp and Follett, 1969 ; Davies and Follett, 1975 ; Ohta and Homma, 1987). In the lesion study, block of the photoperiodic response is effective even though the GnRH system has been left intact (Juss, 1993). In addition, electrical stimulation of IN and IH increases LH secretion (Konishi et al., 1987) and induces testicular growth (Ohta et al., 1984). Furthermore, it has been shown that the expression of Fos-like immunoreactivity in IN, IH and ME by photostimulation of one long day photoperiod (Meddle and Follett, 1995 ; 1997). In addition to these reports, deep brain photoreceptors are thought to be localized in the IN (Silver et al., 1988).

\section{Where is the photoperiodic clock?}

It is an established fact that the circadian clock is involved in the PTM (Pittendrigh, 1972). Circadian $(\sim 24 \mathrm{~h})$ rhythms are a fundamental property of living systems and impose a $24 \mathrm{~h}$ temporal organization regulating the physiology and biochemistry of most organisms. The intracellular clock mechanism is composed by interacting positive and negative transcriptional feedback loops that drive $\sim 24$ hour rhythms in the RNA and protein levels of key clock components called "clock genes" (Dunlap, 1999 ; Reppert and Weaver, 2002). Recently, homologues of mammalian circadian clock genes have been cloned in birds, which have provided a way to examine the molecular link between the circadian clock and photoperiodism in bird (Yoshimura et al., 2000 ; Okano et al., 2001 ; Yamamoto et al., 2001 ; Fu et al., 2002). Avian circadian rhythms are regulated by a multiple oscillatory system consisting of the eye, the pineal gland, and the suprachiasmatic nucleus (SCN) (Ebihara et al., 1987 ; 
Yoshimura et al., 2001). However, enucleation or pinealectomy or lesion around the SCN has no effect on the photoperiodic response of gonads in birds (Davies and Follett, 1975 ; Siopes and Wilson, 1974).

Yasuo et al. (2003) examined the expression of all known avian circadian clock genes (Clock, Per2, Per3, Bmal1, Cry1, Cry2, and E4bp4) in Japanese quail brain and observed all gene expressions in the MBH (Fig. 2). Temporal expression patterns of clock genes in the $\mathrm{MBH}$ were stable under various light conditions. Together with the previous lesion studies, their results suggest that the clock in the $\mathrm{MBH}$ is the longsought clock essential for PTM, and appears to enable animals to retain a steady state photoinducible phase (Yasuo et al., 2003 ; Ball and Balthazart, 2003).

\section{Identification of a gene which is responsible for PTM}

Now it seems that all the molecular machinery required for PTM is localized in the $\mathrm{MBH}$. Since light pulses at photoinducible phase are known to mimic photoperiodically induced testicular growth (Follett and Sharp, 1969 ; Wada, 1979) (Fig. 3), it is

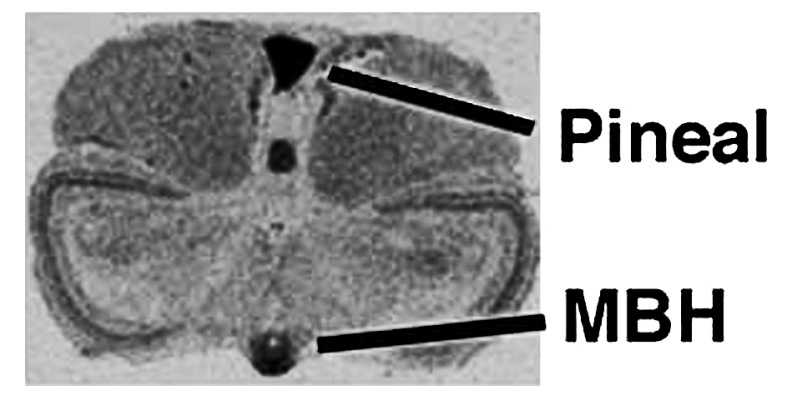

Fig. 2. Expression of circadian clock gene in the mediobasal hypothalamus (MBH) of Japanese quail. Representative autoradiogram for Per2 expression of the coronal section.

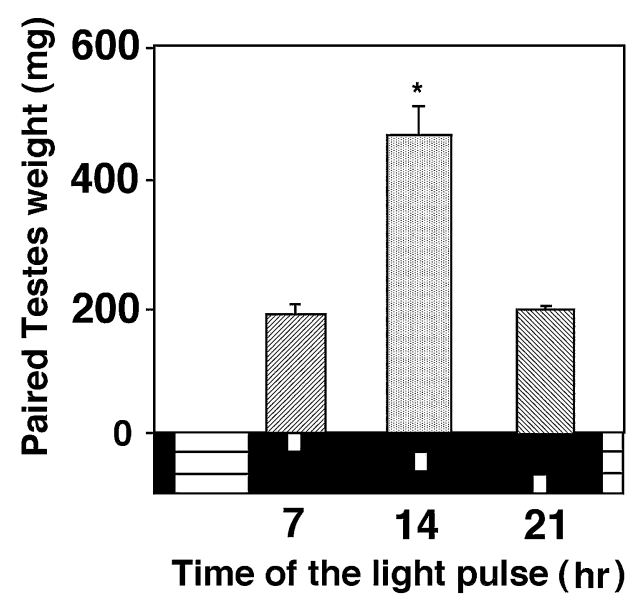

Fig. 3. Light pulses at photoinducible phase cause testicular growth. Animals were given light pulses for 10 days. 
expected that single light pulse at photoinducible phase causes a molecular event in the $\mathrm{MBH}$. To identify the genes that are responsible for the regulation of PTM in birds, Yoshimura et al. (2003) performed a differential subtractive hybridization analysis using $\mathrm{MBH}$ of birds given a light pulse at photoinducible phase versus birds given no light pulse. Among 150 clones, they found only one gene, type 2 iodothyronine deiodinase (Dio2) gene, whose expression was significantly induced by light in the $\mathrm{MBH}$ (Yoshimura et al., 2003). It is noteworthy that expression site of Dio2 in the MBH was entirely consistent with the lesion study (Sharp and Follett, 1969).

\section{Content and target site of thyroid hormone}

Dio2 is an enzyme that converts prohormone thyroxine $\left(\mathrm{T}_{4}\right)$ to active triiodothyronine $\left(\mathrm{T}_{3}\right)$, which is primarily responsible for thyroid hormone action. Dio2 plays an essential role in the local control of brain $T_{3}$ through mechanisms that operate under a variety of situations to maintain $\mathrm{T}_{3}$ concentrations in a narrow range (Bernal, 2002). To determine the level of $T_{3}$ and $T_{4}$ in the $M B H$, Yoshimura et al. (2003) measured the contents of the pooled $\mathrm{MBH}$ collected from 10 animals in each group under short and long day conditions. Although the plasma concentration of $\mathrm{T}_{3}$ and $\mathrm{T}_{4}$ was not significantly different between short and long day conditions, both contents in the $\mathrm{MBH}$ were about 10 fold higher for long days than short days. In addition to these results, they examined the expression of thyroid hormone receptors and found expression of $\operatorname{TR} \alpha, \operatorname{TR} \beta$ and the $\operatorname{RXR} \alpha$ genes in the IN and the ME. These results indicate that locally generated $T_{3}$ acts on the IN and the ME.

\section{Morphological changes in the ME under short and long photoperiods}

It was suggested that photoperiodic GnRH release could be controlled at the GnRH terminals by glia (Dawson et al., 2001 ; Meddle and Follett, 1997), and thyroid hormones have a critical involvement in the development, plasticity and function of the central nervous system (Bernal, 2002). Therefore, to validate the effect of $T_{3}$, Yamamura et al. (2004) examined ultrastructures of GnRH terminals in the ME under long and short day conditions using transmission electron microscopy (TEM). Immunoelectron microscopy revealed that GnRH nerve terminals are in close proximity to the basal lamina in birds subjected to long day conditions. Conventional TEM demonstrated encasement of nerve terminals by the endfeet of glial processes under short days. This morphological change seems to allow the neurons to secrete $\mathrm{GnRH}$ due to the increased access of their terminals to the perivascular area surrounding the fenestrated portal capillaries under long days (Fig. 4). These results support the hypothesis that the photoperiodic response of Japanese quail is regulated primarily at the level of secretion of GnRH rather than by the synthesis of GnRH in the cell bodies (Meddle and Follett, 1997 ; Dawson et al., 2001).

\section{Administration of thyroid hormone induces gonadal growth}

To assess whether $T_{3}$ mediates the photoperiodic response of the gonads, Yoshimura et al. (2003) further studied the effect of the intracerebroventricular (i.c.v.) 

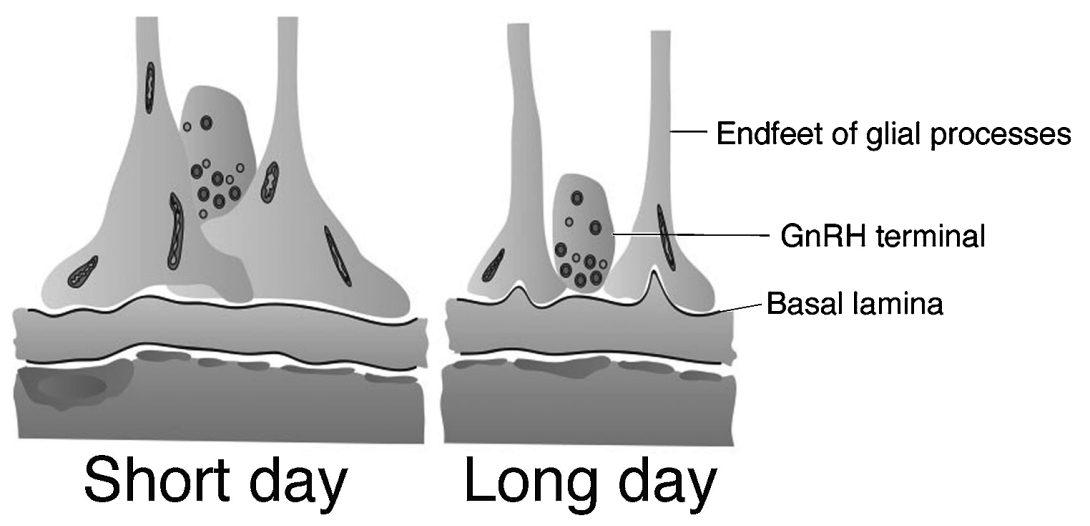

Fig. 4. Schematics of the morphological changes in the GnRH nerve terminals and endfeet of glial processes in the median eminence under short days and long days.

infusion of physiological doses of $T_{3}$ on testicular growth. $T_{3}$ infusion induced testicular growth in a dose dependent manner even though the animals were kept under short day conditions, while $\mathrm{T}_{4}$ infusion had only a minor effect. Interestingly, however, significant testicular growth was not observed in the largest dose of $T_{3}$. Although thyroid hormone is of critical importance in the regulation of photoperiodically induced gonadal growth, this hormone is also known to be somehow involved in the regulation of photorefractoriness (insensitivity to previously stimulatory daylength) (Dawson et al., 2001 ; Dawson and Thapliyal, 2001). The observation that no significant testicular growth is induced at the highest dose of $T_{3}$, suggests the possibility that photorefractoriness occurs as a result of down-regulation of thyroid hormone activity. This hypothesis remains to be clarified in future studies.

It has also been reported that peripheral pharmacological doses of thyroid hormone injection mimics photoperiodically induced gonadal growth. However, in contrast to the central administration, $T_{4}$ is more effective than $T_{3}$ in peripheral administration (Follett et al., 1988 ; Follett and Nicholls 1988). These reports seem to be in contradictory. However, in blood, about $1 / 3$ and $45 \%$ of $\mathrm{T}_{4}$ is known to be converted to $T_{3}$ and reverse $T_{3}\left(r T_{3}\right)$, respectively. In addition, $T_{3}$ is converted to $3,3^{\prime}-T_{2}$ in the blood (Chopra et al., 1978). Therefore, it seems that most of the peripherally delivered $\mathrm{T}_{4}$ might be converted to $\mathrm{T}_{3}$ and act on the central nervous system. The observation that orally administered $\mathrm{T}_{4}$ mimics photoperiodically induced gonadal growth while orally administered $T_{3}$ has no effect further supports this idea (Yasuo et al., $2004 \mathrm{a}$ ).

\section{Decoding photoperiodic information in the pars tuberalis}

Prolactin (PRL) secretion is also regulated by photoperiod. In mammals, the pars tuberalis (PT) in the pituitary is known to be involved in the regulation of photoperiodic regulation of PRL secretion. In birds, however, hypothalamic vasoactive intestinal peptide (VIP) is implicated in PRL secretion, and physiological roles of the avian PT remain unknown. Yasuo et al. (2004 b) have found expression of Per2 and 
Cryl genes in the PT of Japanese quail. The peak of Per2 expression was observed around dawn, while that of Cryl was found around dusk under both long and short photoperiods, resulting in a different phase relationship between the two genes. In mice, protein-protein interactions between PER and CRY affect their stability, nuclear entry and subsequent gene transcription (Lowrey et al., 2000, Shearman et al., 2000). Therefore, changes in the phasing of Per-Cry expression is a potential mechanism by which the long day and short day light signals can be decoded. The level of coincidence in turn determines the formation of PER-CRY protein complexes that can control the transcription of other clock genes and downstream genes that dictate the secretory activity of the prolactin-releasing factor that drives the lactotrophs to secrete PRL (Lincoln et al., 2003 ; Yasuo et al., 2004 b).

\section{Conclusion}

Although thyroid hormone was known to be involved in the regulation of PTM in birds, its precise role had been uncertain for several decades (Dawson, 1993 ; 1998 ; Dawson et al., 2001 ; Dawson and Thapliyal, 2001 ; Follett and Nicholls, 1988 ; Follett et al., 1988). Recent findings have finally determined the target site and molecular events that takes place in the brain.

Finally, it is also of interest to note that multiple studies indicate that thyroid hormones are essential for the maintenance of seasonal reproductive changes in a number of mammals (Nicholls et al., 1988). Recently, it is reported that Dio2 gene is also photoperiodically regulated in the Djungarian hamster, a long day breeder (Watanabe et al., 2004). Thus, recent findings may offer the key to an understanding of the molecular mechanism of PTM in all photoperiodic vertebrates.

\section{Acknowledgement}

This work was supported by the Program for Promotion of Basic Research Activities for Innovative Biosciences (PROBRAIN). Appreciation is expressed to Dr. Shizufumi Ebihara for supporting the study.

\section{References}

Ball GF and Balthazart J. Birds return every spring like clockwork, but where is the clock? Endocrinology, $144:$ 3739-3741. 2003.

Bernal J. Action of thyroid hormone in brain. Journal of Endocrinology Investigation, 25 : 268288. 2002.

Chopra IJ, Solomon DH, Chopra U, Wu SY, Fisher DA and Nakamura Y. Pathways of metabolism of thyroid hormones. Recent Progess in Hormone Research, 34 : 521-567. 1978.

Davies DT and Follet BK. The neuroendocrine control of gonadotrophin release in Japanese quail. I. The role of the tuberal hypothalamus. Proceedings of the Royal Society of London B, $191: 303-315.1975$.

Dawson A. Thyroidectomy progressively renders the reproductive system of starlings (Sturnus vulgaris) unresponsive to changes in daylength. Journal of Endocrinology, $139: 51-55$. 1993.

Dawson A. Thyroidectomy of house sparrow (Passer domesticuls) prevents photo-induced testicular growth but not the increased hypothalamic gonadotrophin-releasing hormone. General and Comparative Endocrinology, $110: 196-200.1998$. 
Dawson A, King VM, Bentley GE, and Ball GF. Photoperiodic control of seasonality in birds. Journal of Biological Rhythms, $16: 365-380.2001$.

Dawson A and Thapliyal JP. The thyroid and photoperiodism. In : Avian Endocrinology (Dawson A and Chaturvedi CM eds.). pp. 141-151. Narosa, New Delhi. 2001.

Dunlap JC. Molecular bases for circadian clocks. Cell, 96 : 271-290. 1999.

Ebihara S, Oshima I, Yamada H, Goto M and Sato K. Circadian organization in the pigeon. In : Comparative Aspects of Circadian Clocks. (Hiroshige T and Honma K. eds.) pp.88-94. Sapporo, Hokkaido University Press. 1987.

Follett BK and Sharp PJ. Circadian rhythmicity in photoperiodically induced gonadotrophin release and gonadal growth in the quail. Nature, $223: 968-971.1969$.

Follett BK and Nicholls TJ. Acute effect of thyroid hormones in mimicking photoperiodically induced release of gonadotropins in Japanese quail. Journal of Comparative Physiology B, $157: 837-843.1988$.

Follett BK, Nicholls TJ and Mayes CR. Thyroxine can mimic photoperiodically induced gonadal growth in Japanese quail. Journal of Comparative Physiology B, 157 : 829-835. 1988.

$\mathrm{Fu} Z$, Inaba M, Noguchi $\mathrm{T}$ and Kato $\mathrm{H}$. Molecular cloning and circadian regulation of cryptochrome genes in Japanese quail (Coturnix coturnix japonica). Journal of Biological Rhythms, $17: 14-27.2002$.

Gwinner E, Hau M and Heigl S. Melatonin : generation and modulation of avian circadian rhythms. Brain Research Bulletin, 44 : 439-444. 1997.

Juss TS. Neuroendocrine and neural changes associated with the photoperiodic control of reproduction. In : Avian Endocrinology (Sharp PJ ed.). pp. 47-60. Society for Endocrinology, Bristol. 1993.

Konishi H, Foster RG and Follett BK. Evidence for a daily rhythmicity in the acute release of LH in response to electrical stimulation in the Japanese quail. Journal of Comparative Physiology A, 161 : 315-319. 1987.

Lincoln GA, Andersson H and Hazlerigg D. Clock genes and the long-term regulation of prolactin secretion : evidence for a photoperiod/circannual timer in the pars tuberalis. Journal of Neuroendocrinology, $15:$ 390-397. 2003.

Lowrey PL, Shimomura K, Antoch MP, Yamazaki S, Zemenides PD, Ralph MR, Menaker M and Takahashi JS. Positional syntenic cloning and functional characterization of the mammalian circadian mutation tau. Science, $288:$ 483-492. 2000.

Meddle SL and Follett BK. Photoperiodic activation of Fos-like immunoreactive protein in neurons within the tuberal hypothalamus of Japanese quail. Journal of Comparative Physiology A, 176, 79-89. 1995.

Meddle SL and Follett BK. Photoperiodically driven changes in Fos expression within the basal tuberal hypothalamus and median eminence of Japanese quail. Journal of Neuroscience, $17:$ : 8909-8918. 1997.

Nicholls TJ, Follett BK, Goldsmith AR and Pearson H. Possible homologies between photorefractoriness in sheep and birds : the effect of thyroidectomy on the length of the ewe's breeding season. Reproduction Nutrition Development, 28 : 375-385. 1988.

Ohta M and Homma K. 1987. Detection of neural connections to the infundibular complex by partial or complete hypothalamic deafferentation in male quail. General and Comparative Endocrinology, $68: 286-292.1987$.

Ohta M, Wada M and Homma K. Induction of rapid testicular growth in quail by phasic electrical stimulation of the hypothalamic photosensitive area. Journal of Comparative Physiology A, 154 : 583-589. 1984.

Okano T, Yamamoto K, Okano K, Hirota T, Kasahara T, Sasaki M, Takanaka Y and Fukada Y. Chicken pineal clock genes : implication of BMAL2 as a bidirectional regulator in circadian clock oscillation. Genes to Cells, $6: 825-836.2001$.

Pittendrigh CS. Circadian surfaces and the diversity of possible roles of circadian organization in photoperiodic induction. Proceedings of National Academy of Sciences USA, 69 : 27342737. 1972.

Reppert SM and Weaver DR. Coordination of circadian timing in mammals. Nature, 418, 935- 
941. 2002.

Sharp PJ and Follett BK. The effect of hypothalamic lesions on gonadotrophin release in Japanese quail (Coturnix coturnix japonica). Neuroendocrinology, 5 : 205-218. 1969.

Shearman LP, Sriram S, Weaver DR, Maywood ES, Chaves I, Zheng B, Kume K, Lee CC, van der Horst GT, Hastings MH and Reppert SM. Interacting molecular loops in the mammalian circadian clock. Science, 288 : 1013-1019. 2000.

Silver R, Witkovsky P, Horvath P, Alones V, Barnstable CJ, Lehman MN. Coexpression of opsinand VIP-like-immunoreactivity in CSF-contacting neurons of the avian brain. Cell and Tissue Research, 253 : 189-198. 1988.

Siopes TD and Wilson WO. Extraocular modification of photoreception in intact and pinealectomized coturnix. Poultry Science, 53 : 2035-2041. 1974.

Wada M. Photoperiodic control of LH secretion in Japanese quail with special reference to the photoinducible phase. General and Comparative Endocrinology, 39 : 141-149. 1979.

Watanabe M, Yasuo S, Watanabe T, Yamamura T, Nakao N, Ebihara S and Yoshimura T. Photoperiodic regulation of type 2 deiodinase gene in Djungarian hamster : Possible homologies between avian and mammalian photoperiodic regulation of reproduction. Endocrinology, $145: 1546-1549$.

Yamamoto K, Okano T and Fukada Y. Chicken pineal Cry genes : light-dependent up-regulation of cCry1 and cCry2 transcripts. Neuroscience Letters, 313 : 13-16. 2001.

Yamamura T, Hirunagi K, Ebihara S and Yoshimura T. Seasonal morphological changes in the neuro-glial interaction between GnRH nerve terminals and glial endfeet in Japanese quail. Endocrinology, 145 : 4264-4267. 2004.

Yasuo S, Watanabe M, Okabayashi N, Ebihara S and Yoshimura T. Circadian clock genes and photoperiodism : Comprehensive analysis of clock genes expression in the mediobasal hypothalamus, the suprachiasmatic nucleus and the pineal gland of Japanese quail under various light schedules. Endocrinology, 144 : 3742-3748. 2003.

Yasuo S, Ebihara S and Yoshimura T. Oral thyroxine administration mimics photoperiodically induced gonadal growth in Japanese quail. Animal Science Journal, in press. 2004 a.

Yasuo S, Watanabe M, Tsukada A, Takagi T, Iigo M, Shimada K, Ebihara S and Yoshimura T. Photoinducible phase specific light induction of Cryl gene in the pars tuberalis of Japanese quail. Endocrinology, 145 : 1612-1616. 2004 b.

Yoshimura T, Suzuki Y, Makino E, Suzuki T, Kuroiwa A, Matsuda Y, Namikawa T and Ebihara S. Molecular analysis of avian circadian clock genes. Molecular Brain Research, 78 : $207-$ 215. 2000.

Yoshimura T, Yasuo S, Suzuki Y, Makino E, Yokota Y and Ebihara S. Identification of the suprachiasmatic nucleus in birds. American Journal of Physiology, 280 : R1185-1189. 2001.

Yoshimura T, Yasuo S, Watanabe M, Iigo M, Yamamura T, Hirunagi K and Ebihara S. Light-induced hormone conversion of $\mathrm{T}_{4}$ to $\mathrm{T}_{3}$ regulates photoperiodic response of gonads in birds. Nature, 426 : 178-181. 2003. 